\title{
Manipulation of spin state of iron porphyrin by chemisorption on magnetic substrates
}

\author{
Sumanta Bhandary, ${ }^{1}$ Barbara Brena, ${ }^{1}$ Pooja M. Panchmatia, ${ }^{2}$ Iulia Brumboiu, ${ }^{1}$ Matthias Bernien, ${ }^{3}$ Claudia Weis, ${ }^{4}$ \\ Bernhard Krumme, ${ }^{4}$ Corina Etz, ${ }^{1}$ Wolfgang Kuch, ${ }^{3}$ Heiko Wende, ${ }^{4}$ Olle Eriksson, ${ }^{1}$ and Biplab Sanyal ${ }^{1, *}$ \\ ${ }^{1}$ Department of Physics and Astronomy, Uppsala University, Box 516, 75120 Uppsala, Sweden \\ ${ }^{2}$ School of Applied Sciences, University of Huddersfield, Queensgate, Huddersfield HD1 3DH, United Kingdom \\ ${ }^{3}$ Institut für Experimentalphysik, Freie Universität Berlin, Arnimallee 14, 14195 Berlin, Germany \\ ${ }^{4}$ Faculty of Physics and Center for Nanointegration Duisburg-Essen (CeNIDE), University of Duisburg-Essen, \\ Lotharstrasse 1, 47048 Duisburg, Germany
}

(Received 29 October 2012; revised manuscript received 17 May 2013; published 1 July 2013)

\begin{abstract}
One of the key factors behind the rapid evolution of molecular spintronics is the efficient realization of spin manipulation of organic molecules with a magnetic center. The spin state of such molecules may depend crucially on the interaction with the substrate on which they are adsorbed. In this paper we demonstrate, using $a b$ initio density functional calculations, that the stabilization of a high spin state of an iron porphyrin (FeP) molecule can be achieved via chemisorption on magnetic substrates of different species and orientations, viz., $\mathrm{Co}(001), \mathrm{Ni}(001), \mathrm{Ni}(110)$, and $\mathrm{Ni}(111)$. The signature of chemisorption of $\mathrm{FeP}$ on magnetic substrates is evident from broad features in $\mathrm{N} K$ x-ray absorption (XA) and $\mathrm{Fe} L_{2,3}$ x-ray magnetic circular dichroism (XMCD) measurements. Our theoretical calculations show that the strong covalent interaction with the substrate increases Fe-N bond lengths in FeP and hence a switching to a high spin state $(S=2)$ from an intermediate spin state $(S=1)$ is achieved. Due to chemisorption, ferromagnetic exchange interaction is established through a direct exchange between $\mathrm{Fe}$ and substrate magnetic atoms as well as through an indirect exchange via the $\mathrm{N}$ atoms in FeP. The mechanism of exchange interaction is further analyzed by considering structural models constructed from $a b$ initio calculations. Also, it is found that the exchange interaction between $\mathrm{Fe}$ in $\mathrm{FeP}$ and a Ni substrate is almost 4 times smaller than with a Co substrate. Finally, we illustrate the possibility of detecting a change in the molecular spin state by XMCD, Raman spectroscopy, and spin-polarized scanning tunneling microscopy.
\end{abstract}

DOI: 10.1103/PhysRevB.88.024401

PACS number(s): 71.70.Gm, 34.35.+a, 68.43.Bc, 74.25.nd

\section{INTRODUCTION}

Manipulation and detection of spin states of organic molecules with a magnetic center are important issues in molecular spintronics. The change in the spin state can be achieved by external agents like temperature, light, pressure, etc. Recently it was shown ${ }^{1}$ that a transition from $S=1$ to $S=2$ spin state of an iron porphyrin $(\mathrm{FeP})$ molecule is possible by strain engineering of a defected graphene sheet. The microscopic mechanism behind this change in the spin state is identified as the change in $\mathrm{Fe}-\mathrm{N}$ bond length due to the interaction with strained graphene. The difference in the occupancy of molecular orbitals due to different bond lengths is responsible for the change in the magnetic moment of the $\mathrm{Fe}$ atom at the center of FeP. In this paper we demonstrate another route to realize this effect, now by chemisorption on a magnetic metallic substrate, which may offer an easier experimental investigation.

Metalorganic molecules supported on magnetic substrates have attracted a lot of attention in the last few years. ${ }^{2}$ The primary focus is to understand and manipulate magnetic exchange coupling between the $3 d$ metal center of a metalorganic [iron porphyrin $(\mathrm{FeP})$, iron phthalocyanine $(\mathrm{FePc})$, cobalt phthalocyanine $(\mathrm{CoPc})$, etc.] molecule and a metallic magnetic substrate, such as $\mathrm{Co}, \mathrm{Ni}$, etc. It has been shown that the exchange coupling can be tuned to be either ferromagnetic ${ }^{3}$ or antiferromagnetic, ${ }^{4}$ depending on the chemical environment and hence on the mechanism of magnetic exchange interaction between $\mathrm{Fe}$ and the magnetic atom of the substrate. Sophisticated experimental tools, such as element-specific x-ray magnetic circular dichroism (XMCD) ${ }^{3-6}$ and spin-polarized scanning tunneling microscopy (SP-STM) ${ }^{7}$ as well as accurate density functional theory calculations ${ }^{3-5,7}$ have played an instrumental role in uncovering the microscopic mechanisms behind these exotic phenomena.

Adsorption of metalorganics, e.g., FeP, on a metallic magnetic substrate can take place in two distinct ways. One is physisorption, where the molecule has a weak van der Waals interaction with the substrate without forming any chemical bond and hence, more or less preserves its gas phase properties. However, in the scenario of chemisorption, the molecule-substrate interaction is much stronger in the presence of well established chemical bonds. ${ }^{8}$ A substantial change in the geometry and electronic structure of the molecule is expected in this case due to the change in the ligand field exerted on the $\mathrm{Fe} d$ orbitals via stronger hybridization with substrates. To be precise, the microscopic reason is the enhancement of $\mathrm{Fe}-\mathrm{N}$ bond lengths $(>2.04 \AA)$, which are larger than those in gas phase or the physisorbed molecule. A schematic picture showing this effect is presented in Fig. 1. Moreover, we will show below that irrespective of surface orientation, FeP prefers to stabilize itself in such a way that it maximizes the chemical bonding between the nitrogen atoms of FeP and the underlying substrate atoms. Hence, we propose a general procedure of stabilizing metalorganics on magnetic substrates via chemisorption to introduce a mechanical strain on the molecule which leads to a transition from a low to a high spin state. We will also show how one can detect the change in the spin states by Raman spectroscopy and SP-STM experiments. 


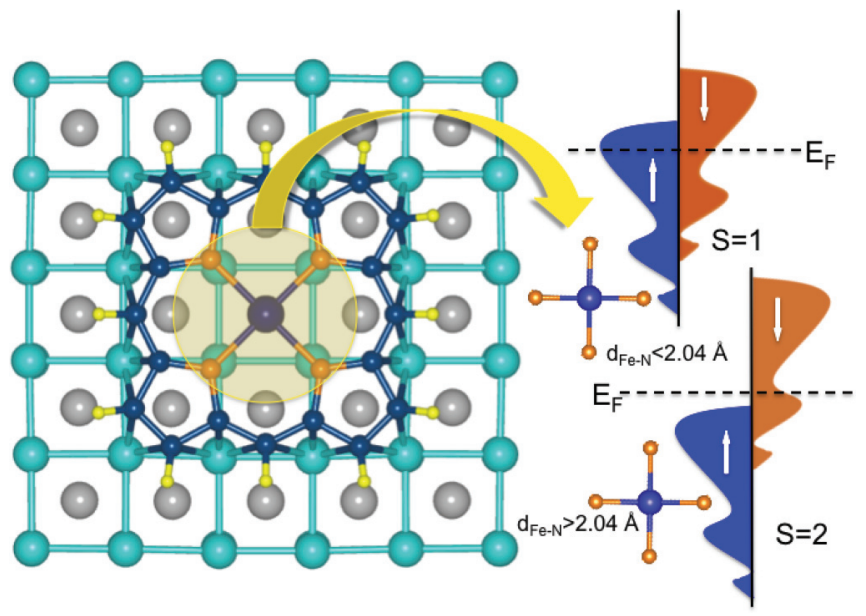

FIG. 1. (Color online) Schematic picture of the geometry of a $\mathrm{FeP}$ molecule on a $\mathrm{Co}(001)$ substrate. The turquoise and gray balls represent $\mathrm{Co}$ atoms of the first and second layers of the substrate, respectively. $\mathrm{C}$ atoms are shown in steel, while $\mathrm{Fe}, \mathrm{N}$, and $\mathrm{H}$ atoms are represented with blue, orange, and yellow colors, respectively. The shaded central part demonstrates the change in the Fe-N bond distance along with the change in the spin state. A smaller Fe-N bond length $(<2.04 \AA)$ leads to a smaller exchange splitting with a $S=1$ spin state (top right). Both the majority and minority spin channels are thus partially filled. A larger exchange splitting is observed for an increased Fe-N bond length ( $>2.04 \AA$ ), where the majority channel gets occupied completely and a high spin state $(S=2)$ is obtained (bottom right).

\section{COMPUTATIONAL AND EXPERIMENTAL DETAILS}

We have performed first-principles density functional calculations using the VASP code. ${ }^{9,10}$ Plane wave projector augmented wave basis was used in the Perdew-Burke-Ernzerhof generalized gradient approximation (PBE-GGA) for the exchange correlation potential. The plane wave cutoff energy used was $400 \mathrm{eV}$. A $3 \times 3 \times 1$ Monkhorst Pack $k$ point set was used for the integration in the Brillouin zone. Atoms were relaxed until the Hellman-Feynman forces were minimized up to $0.01 \mathrm{eV} / \AA$. We have used $7 \times 7$ lateral supercells of the magnetic substrate for the (001) and (111) orientations and a $6 \times 7$ supercell for the (110) surfaces. In all cases, a slab of three layers and $21 \AA$ of vacuum perpendicular to the substrate were considered. For geometry optimizations, the atoms in the lowest layer were kept fixed and all the other atoms in the slab as well as in the FeP molecule were allowed to relax. To account for the electronic correlations in the narrow $d$ states of Fe in the FeP molecule, we have used a GGA $+U$ approach, where a Coulomb interaction term is added according to the mean field Hubbard $U$ formalism. ${ }^{11}$ The values of the Coulomb parameter $U$ and the exchange parameter $J$ were chosen to be 4 and $1 \mathrm{eV}$, respectively, as these values correctly reproduce the electronic structure and magnetism of FeP in the gas phase. ${ }^{12}$ For all cases, we have used a semiempirical approach ${ }^{13}$ to account for van der Waals interaction between FeP and the substrates.

X-ray absorption (XA) and XMCD measurements have been performed at the beamline UE56/2 PGM2 at BESSY II. Linearly $p$-polarized $\mathrm{x}$ rays with $150 \mathrm{meV}$ energy resolution were used at the $\mathrm{N} K$ edge. Circularly polarized $X$ rays with $90 \%$ polarization degree and $300 \mathrm{meV}$ energy resolution were employed at the $\mathrm{Fe}$ and $\mathrm{Ni} L_{2,3}$ edges. Spectra were recorded by total electron yield measurements and normalized to a gold grid reference signal as well as to the corresponding spectra of the substrate without adsorbed molecules. Sample preparation and measurements were carried out in ultrahigh vacuum with a base pressure of $2 \times 10^{-10}$ mbar. The $\mathrm{Cu}(001)$ substrate was prepared by repeated sputtering and annealing cycles. The Ni was deposited by means of electron beam epitaxy. Fe 2,3,7,8,12,13,17,18-octaethylporphyrin chloride molecules were purchased from porphyrin systems and deposited from a Knudsen cell at about $490 \mathrm{~K}$ onto the substrate held at room temperature. The bulk reference sample was prepared by stamping the molecular powder into an indium foil.

\section{RESULTS AND DISCUSSIONS}

\section{A. Optimized structures}

In this section we provide detailed structural data obtained from our calculations. The generality of the change in molecular spin state has been studied by having different types of substrates and with different orientations, viz., (001), (110), and (111). Magnetic substrates such as Co and $\mathrm{Ni}$ with partially filled $d$ orbitals are chosen to enhance the probability of chemisorption and also to allow for a study of the mechanism of magnetic exchange interactions. Different surface orientations are chosen to provide different crystallographic environments to the molecule. Ni surfaces with hexagonal (111), rectangular (110), and square (001) facets are considered along with a $\mathrm{Co}(001)$ surface. The (100) surface orientation of $\mathrm{Co}$ and $\mathrm{Ni}$ provides the most symmetric substrate (square) for the molecule, whereas the rectangular (110) or hexagonal (111) surface provides a less symmetric environment. As we will see below, this affects the stabilization as well as the geometry of the chemisorbed FeP molecule. To be more specific, on a square substrate, $\mathrm{Co} / \mathrm{Ni}$ atoms interact with all four $\mathrm{N}$ atoms of $\mathrm{FeP}$ in a similar fashion, whereas on a rectangular surface, the chemical interaction along two perpendicular directions are different. This is true for the hexagonal surface as well. We have also considered a configuration where $\mathrm{FeP}$ is rotated by $45^{\circ}$ with respect to the substrate in order to have a less direct interaction between FeP (predominantly with ligand $\mathrm{N}$ ) and the first substrate layer. Even though we obtain a high spin state for all chemisorbed situations, chemical stabilization depends on the rotation of the molecule with respect to the substrate, as will be discussed below. It is indeed observed that the maximization of the molecule-substrate direct chemical interaction increases the stability of adsorption. In all studied systems, one can observe that the interaction between $\mathrm{N}$ states of the FeP molecule and the surface orbitals of the substrate is maximized in the ground state configuration.

As stated above, stability, spin state, and magnetic interaction of FeP with the surfaces depend largely on the surface orientation as well as the orientation of the molecule itself with respect to the surface underneath. To demonstrate the effects of orientations and resulting bonding situations, we will focus on the configurations of $\mathrm{FeP}$ adsorbed on $\mathrm{Co} / \mathrm{Ni}$ surface with 
(a)

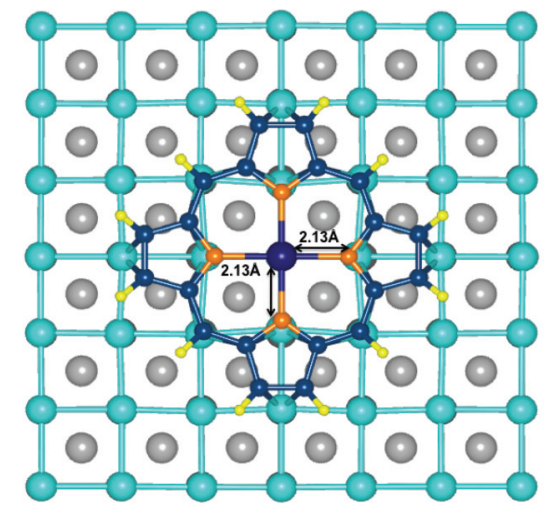

(b)

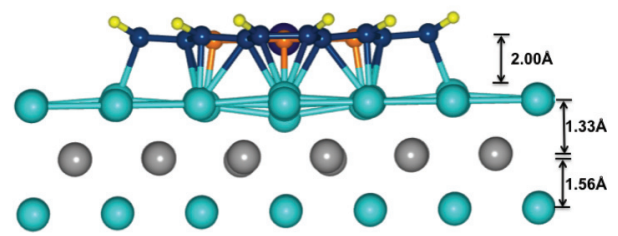

FIG. 2. (Color online) FeP molecule on $\mathrm{Co}(001)$, TOP position. (a) Top view, where Fe-N bond lengths are shown. (b) Side view, where minimum distances between different layers are shown.

different facets. In the following figures, only chemical bonds with distance less than $2.5 \AA$ are shown to make the main content clear.

(i) TOP (001): FeP can be chemisorbed in such a way that the Fe center sits exactly on top of a (001) surface Co atom forming a direct bond between them. The four N's bonded to $\mathrm{Fe}$ also orient themselves such that each of them makes direct chemical bonds with one Co atom on the surface. We denote this particular geometry as the TOP configuration (Fig. 2). The Fe-N bond length in the FeP-gas phase molecule is $2.0 \AA$, which is to be compared to a Co-Co nearest neighbor distance of $2.5 \AA$. To form a direct Fe-Co bond, as well as a N-Co bond, which helps in stability, the molecule has to stretch. Therefore, our calculation demonstrates that the Fe-N bond length is stretched up to $2.13 \AA$ (Fig. 2) when adsorbed on the (001) surface. Moreover, a distortion is observed mostly in the surface layer of Co where the Co-Co bond length is contracted to $2.44 \AA$ close to the site of molecular adsorption. Thus mutual stretching and contraction occur during the formation of the chemical bonds. As the Fe-N and Co-Co bonds do not have exactly the same length, an $\mathrm{Fe}-\mathrm{N}-\mathrm{Co}$ bond angle larger than $90^{\circ}$ is established, $97.44^{\circ}$ to $98.16^{\circ}$, to be precise. The Fe-Co bond length is $2.32 \AA$ even though the minimum distance of the molecule plane to the surface layer is $2.0 \AA$, as shown in Fig. 2(b). The reason behind the discrepancy is that the $\mathrm{Co}$ atom, just below $\mathrm{Fe}$ is pushed down. This also decreases the minimum distance between surface and subsurface Co layer down to $1.33 \AA$, while the minimum distance between the second and third Co layers remains much higher, $1.56 \AA$ [Fig. 2(b)]. It should be noted that a similar geometry and bond distances are observed for $\mathrm{FeP}$ on the $\mathrm{Ni}(001)$ surface (figure not shown).

$T O P-R$ (001): Another possible configuration with $\mathrm{Fe}$ on top of a Co surface atom is denoted as a TOP-R configuration (not shown), where FeP is rotated by $45^{\circ}$ around the vertical Fe-Co axis. In this situation, N's are placed at hollow sites. This (a)

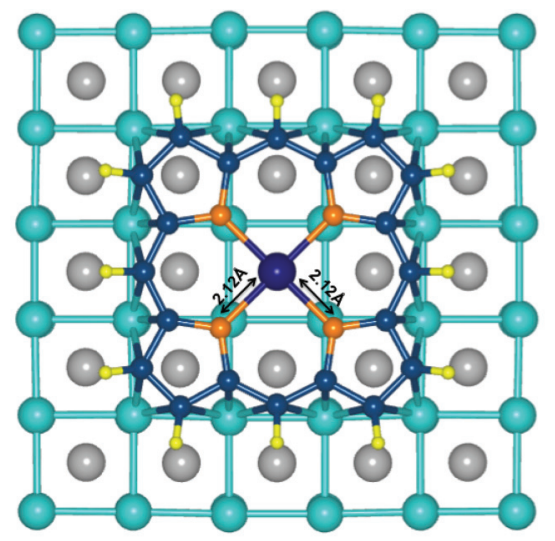

(b)

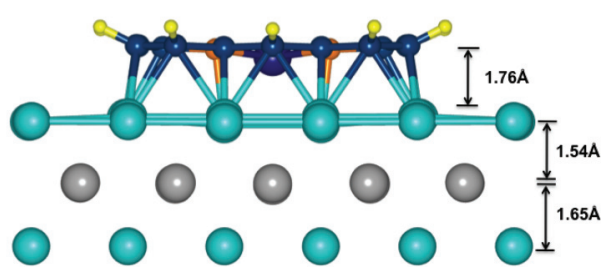

FIG. 3. (Color online) HOLLOW configuration on the $\mathrm{Co}(001)$ surface. (a) and (b) Same convention as in Fig. 2.

prevents the formation of $\mathrm{Co}-\mathrm{N}$ direct bonding and hence, the $\mathrm{Fe}-\mathrm{N}-\mathrm{Co}$ indirect exchange path becomes ineffective.

(ii) HOLLOW (001): The distortion in Co layers is considerably less in the HOLLOW configuration depicted in Fig. 3. In this case, the geometry is stabilized in such a way that the Fe center does not sit on top of any Co atom but on a hollow site, i.e, on top of a second layer Co atom. As there is no direct bonding between $\mathrm{Fe}$ and $\mathrm{Co}$ in this case, the surface Co layer is not affected much. The Fe-Co bond length is much larger here, whereas a direct bond exists between N's and surface $\mathrm{Co}$ atoms. In this case, the next-nearest neighbor Co-Co distance (3.60 $\AA$ ) should be compared with the N-Fe-N distance $(4.25 \AA$ ) [Fig. 3(a)]. This forces the Fe-N-Co bond angle to be $75.35^{\circ}$, which is much smaller than $90^{\circ}$. The minimum distance between FeP and the surface layer is $1.78 \AA$. The minimum distance between the first and second and the second and third layers do not differ much (1.62 and $1.66 \AA$, respectively), as shown in Fig. 3(b).

HOLLOW-R (001): If we rotate FeP with respect to the $H O L L O W$ configuration by $45^{\circ}$ about the vertical axis (denoted as $H O L L O W-R$, not shown here), direct Co-N bonds will not form as in the previous structure. In this case, the Fe-Co bond is longer compared to that in the TOP/TOP-R configuration. Unlike the previous situations, the indirect Fe-N-Co exchange path is ineffective here.

(iii) $T O P-R$ (110): The most stable configuration on the (110) facet of $\mathrm{Ni}$ is $T O P-R$ although the molecule moves a bit from the top site and stabilizes on the bridge site as shown in Fig. 4. Due to the asymmetric nature of the surface, Fe-N bond lengths are different, having a range from 2.07 to $2.17 \AA$. Also FeP loses its flat structure with the minimum distance from the surface being $1.80 \AA$ A. Fe makes bonds with two Ni atoms with bond lengths 2.45 and $2.49 \AA$. Two of the four $\mathrm{N}$ atoms also make direct bonds with surface $\mathrm{Ni}$ atoms. The Fe-N-Ni angles 
(a)

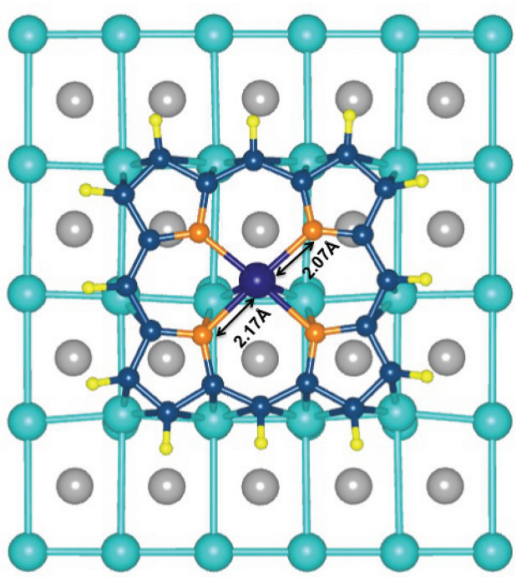

(b)

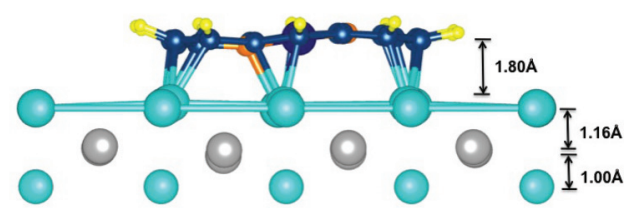

FIG. 4. (Color online) TOP-R configuration on the Ni(110) surface. (a) and (b) Same convention as in Fig. 2.

are $70.85^{\circ}$ and $72.41^{\circ}$. The minimum distances between the first and second and the second and third layers are smaller (1.16 and $1.0 \AA$ ) compared to other cases described before.

(iv) TOP (111): The only configuration that is considered on top of a Ni(111) surface is the TOP one. The underneath hexagonal symmetry in $\mathrm{Ni}$ provides a most asymmetric environment for a square planar Fe-N block in FeP. The Fe-N bonds are thus quite different and vary from 2.04 to $2.15 \AA$ (Fig. 5). Also here, the molecule moves a bit from the top position in such a way that FeP-substrate bonds (specially $\mathrm{N}-\mathrm{Ni}$ bonds) are maximized. The Fe-Ni bond length becomes $2.42 \AA$. Following the asymmetric behavior of the Fe-N bond lengths, the Fe-N-Ni bond angles $\left(92.88^{\circ}, 76.00^{\circ}\right.$, and $\left.82.33^{\circ}\right)$ also differ from each other. The minimum separation of FeP from the substrate is $1.92 \AA$ with a slight distortion in the topmost layer. Finally, the first two interlayer distances in the Ni slab are calculated to be 1.85 and $1.97 \AA$, respectively.

The scenario of chemisorption of FeP on magnetic substrates is manifested indirectly through element-specific spectroscopic measurements. We show here XA and XMCD data for 0.6 monolayers (ML) of Fe octaethylporphyrin (FeOEP) molecules adsorbed on $6 \mathrm{ML}$ of $\mathrm{Ni}$ on $\mathrm{Cu}(001)$. FeOEP has the same structure as FeP except that 8 out of the 12 terminating hydrogen atoms are replaced by ethyl groups. Figure 6(a) shows XA spectra at the $\mathrm{N} K$ edge recorded with linearly $p$-polarized $\mathrm{x}$ rays for three different incidence angles together with the spectrum of a polycrystalline FeOEP bulk reference sample. The angle dependence of the XA spectra reveals that the molecules are flatly adsorbed on the Ni surface. At $55^{\circ}$ incidence (magic angle) the isotropic spectrum is obtained, which is independent of the orientation of the molecules. The spectrum of the bulk sample comprises of two main $\pi^{*}$ resonances at about 398.8 and $401.6 \mathrm{eV}$ and several $\sigma^{*}$ resonances above $404 \mathrm{eV}$. The spectra of the adsorbed (a)

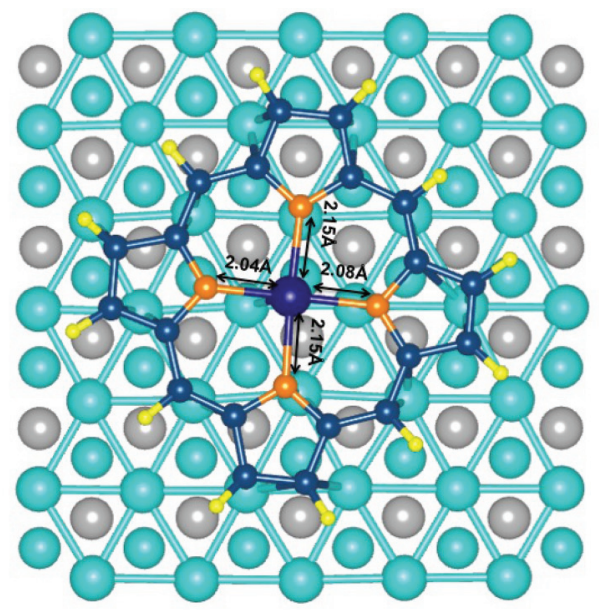

(b)

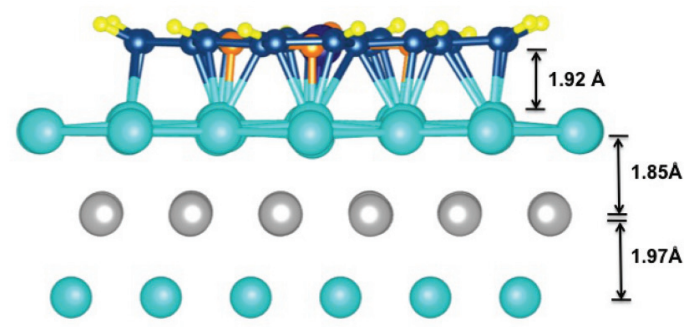

FIG. 5. (Color online) TOP configuration on the Ni(111) surface. (a) and (b) Same convention as in Fig. 2.

molecules show similar features but all resonances are much broader and the $\pi^{*}$ double peak gets slightly compressed. The broadening of the spectroscopic features directly reflects the modification of the $\mathrm{N}$ orbitals due to hybridization with $\mathrm{Ni}$ atoms. ${ }^{14}$ It therefore shows that the molecules are chemisorbed on the surface. In Figs. 6(b) and 6(c) Fe $L_{2,3}$ XA and XMCD spectra of the adsorbed molecules are shown. The spectra have been recorded at $20^{\circ}$ grazing incidence in remanence of the in-plane magnetized $\mathrm{Ni}$ film at a temperature of $43 \mathrm{~K}$. The corresponding $\mathrm{Ni} L_{2,3}$ XMCD spectrum is presented in Fig. 6(d). The presence of an Fe $L_{2,3}$ XMCD signal shows that the Fe magnetic moment of the molecules is aligned by the Ni film. Since the Fe XMCD has the same sign as the $\mathrm{Ni}$ $\mathrm{XMCD}$, the coupling between the molecules and the substrate is ferromagnetic.

\section{B. Exchange interaction and electronic structure}

The chemisorbed FeP is found to undergo a mechanical strain, as detailed above, originating from the molecule-surface interaction, primarily by the $\mathrm{N}-\mathrm{Ni} / \mathrm{N}-\mathrm{Co}$ direct chemical bonding, which leads to a stretching of the $\mathrm{Fe}-\mathrm{N}$ bond lengths of $\mathrm{FeP}$. The stretching can be symmetric [e.g., for a (001) surface] or asymmetric [e.g., for a (110) surface] along different in-plane crystallographic directions depending on the orientation of the surface underneath. Nevertheless, all the stretched geometries yield a high spin state $(S=2)$ of FeP by having the individual Fe-N bond length exceeding $2.04 \AA$. This structural change in FeP has a direct impact on its electronic properties. The four $\mathrm{N}$ atoms bonded to the central $\mathrm{Fe}$ atom (in a $\mathrm{Fe}^{2+}$ state) provide a square planar crystal field. The $p-d$ hybridization 

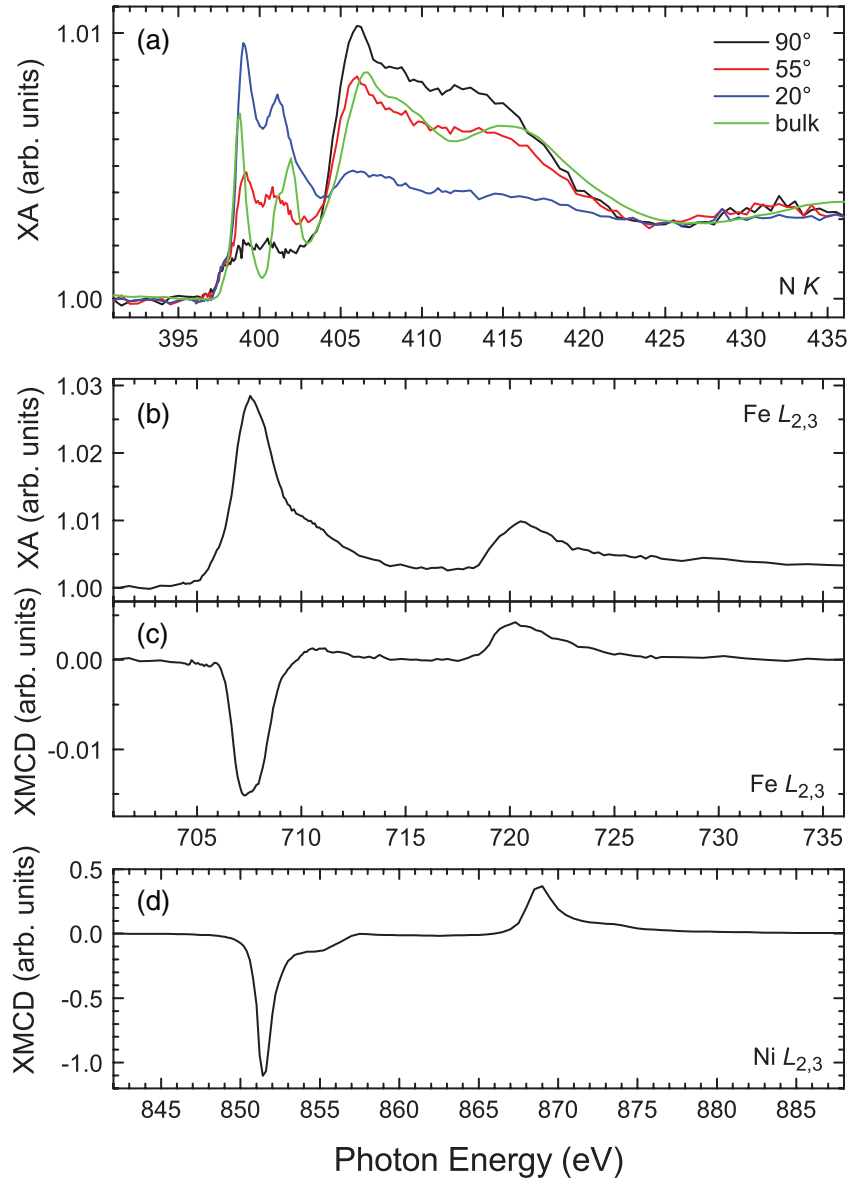

FIG. 6. (Color online) $\mathrm{N} K \mathrm{XA}$ (a), Fe $L_{2,3}$ XA (b), XMCD (c), and Ni $L_{2,3}$ XMCD (d) spectra of 0.6 ML Fe OEP on $6 \mathrm{ML}$ $\mathrm{Ni} / \mathrm{Cu}(001)$. The spectra presented in (a) are measured at room temperature with linearly $p$-polarized $\mathrm{x}$ rays and incidence angles of $90^{\circ}, 55^{\circ}$, and $20^{\circ}$ between the $k$ vector and the surface. The spectrum of a polycrystalline reference sample is shown in green. The spectra presented in (b) to (d) are measured at $T=43 \mathrm{~K}$ with circularly polarized $\mathrm{x}$ rays and $20^{\circ}$ grazing incidence.

between $\mathrm{N}-2 p$ and Fe-3d orbitals shifts the $d_{x^{2}-y^{2}}$ level higher up in energy due to strong $\sigma$-type hybridization, whereas other orbitals feel a weak $\pi$-type hybridization and are less influenced by the interaction with surface states. Along with this covalent contribution, a point charge contribution from $\mathrm{N}$ atoms shifts the $d_{x^{2}-y^{2}}$ level up due to a strong Coulomb repulsion. Both these parts contribute to the ligand field (LF) effects which are strong when the Fe-N bond length is smaller, e.g., in the gas phase. Hence, the LF dominates over the intra-atomic exchange and an intermediate spin state $(S=1)$ is observed with four spin-up and two spin-down electrons. As the Fe-N bond length is stretched (Fig. 1), the LF strength decreases resulting in the lowering of the $d_{x^{2}-y^{2}}$ orbital energy. The electrons now occupy Fe-3d orbitals following Hund's first rule by filling up all majority spin levels followed by one level in the minority spin channel. Even though we have observed a strong bonding between $\mathrm{Fe} d_{z^{2}}$ and a surface atom, as the spin state change is mostly governed by the shift of the $d_{x^{2}-y^{2}}$ orbital, LF effects explain the spin state of the chemisorbed FeP molecule.
TABLE I. Calculated exchange energies $\left(E^{\mathrm{ex}}=E^{\mathrm{AFM}}-E^{\mathrm{FM}}\right)$ and relative energies with respect to the ground state (in parentheses, with the ground state indicated in bold) in $\mathrm{eV}$ are shown for different substrates and their orientations. Note that the positive $E^{\mathrm{ex}}$ indicates that the FM alignment is stable. The configurations denoted by TOP, TOP-R, HOLLOW, and HOLLOW-R are defined in the text. For the $\mathrm{Ni}(111)$ surface, the TOP configuration is the only relevant one.

\begin{tabular}{lcccc}
\hline \hline Surface & TOP & TOP-R & HOLLOW & HOLLOW-R \\
\hline $\mathrm{Co}(001)$ & $0.32(2.49)$ & $0.51(3.6)$ & $\mathbf{0 . 7 3}(\mathbf{0 . 0})$ & $0.33(4.38)$ \\
$\mathrm{Ni}(001)$ & $0.07(2.17)$ & $0.2(3.58)$ & $\mathbf{0 . 1 6}(\mathbf{0 . 0})$ & $0.10(3.13)$ \\
$\mathrm{Ni}(110)$ & $0.04(2.5)$ & $\mathbf{0 . 0 9}(\mathbf{0 . 0})$ & $0.43(1.06)$ & $0.13(1.88)$ \\
$\mathrm{Ni}(111)$ & 0.13 & - & - & - \\
\hline \hline
\end{tabular}

Due to chemisorption, the Fe moment has a strong exchange interaction with the substrate $\mathrm{Co} / \mathrm{Ni}$ moments. The magnetic exchange coupling energies $\left(E^{\mathrm{ex}}=E^{\mathrm{AFM}}-E^{\mathrm{FM}}\right)$ obtained in the ground state structures on each surface are given in Table I. FM and AFM denote ferromagnetic and antiferromagnetic alignments between the $\mathrm{Fe}$ and substrate moments, respectively. It should be noted that both direct and indirect superexchange interaction give rise to a ferromagnetic exchange coupling in these systems. When both $\mathrm{Fe}$ and $\mathrm{N}$ atoms are on top of surface atoms, a $(\mathrm{Co} / \mathrm{Ni})-\mathrm{Fe}$ direct exchange as well as $(\mathrm{Co} / \mathrm{Ni})-\mathrm{N}-\mathrm{Fe}$ indirect superexchange interactions are operational. This situation is denoted as the TOP configuration, for which spin-polarized DOS, energyresolved charge, and magnetization densities are shown for the $\mathrm{Co}(001)$ surface in Fig. 7. The charge density is plotted [inset of Fig. 7(a)] in an energy window [shaded part in Fig. 7(a)] of $0.23 \mathrm{eV}$ where the hybridization between $\mathrm{Fe}-\mathrm{N}, \mathrm{N}-\mathrm{Co}$, and $\mathrm{Fe}-\mathrm{Co}$ orbitals are dominant. The cross section of the total charge density in the (100) plane [Fig. 7(a)] indicates a substantial overlap between the orbitals mentioned above. The magnetization densities shown in Figs. 7(b) and 7(c) indicate a similar overlap between in-plane and out-of-plane orbitals carrying magnetic moments. To be precise, the $d_{x^{2}-y^{2}}$ orbital of $\mathrm{Fe}$ hybridizes with the orbitals of $\mathrm{N}$ in the plane of $\mathrm{FeP}$ as seen in the spin-down channel [Fig. 7(b)]. The out-of-plane hybridization between Fe-Co and N-Co via $d_{z^{2}}$ and $p_{z}$ orbitals is observed in the spin-up channel [Fig. 7(c)]. Therefore, the charge and magnetization densities show the signatures of a direct exchange as well as an indirect superexchange interaction between $\mathrm{Fe}$ and substrate $\mathrm{Co}$ atoms.

As mentioned above, $\mathrm{FeP}$ can be adsorbed in other ways, e.g., TOP-R, HOLLOW, and HOLLOW-R configurations. If $\mathrm{FeP}$ is rotated by $45^{\circ}$ with respect to the substrate (denoted as TOP-R/HOLLOW-R), $\mathrm{N}$ atoms are no longer placed on top of substrate atoms. In this situation the effect of indirect superexchange is minimized and the direct exchange between the $\mathrm{Fe}$ atom in $\mathrm{FeP}$ and the substrate atoms dominates. But the absence of $\mathrm{N}$-substrate bonds pushes the molecule further apart from the substrate which, in turn, has an effect on the direct exchange. On the $\mathrm{Co}(100)$ surface, FM exchange is favored over AFM by $0.39 \mathrm{eV}$ for the TOP configuration and by $0.49 \mathrm{eV}$ for TOP-R configuration. For the HOLLOW configuration, which is the most favorable one, the exchange coupling energy is $0.75 \mathrm{eV}$, indicating a strong FM interaction. Also, it is seen from Table I that the calculated coupling energy 
(a)

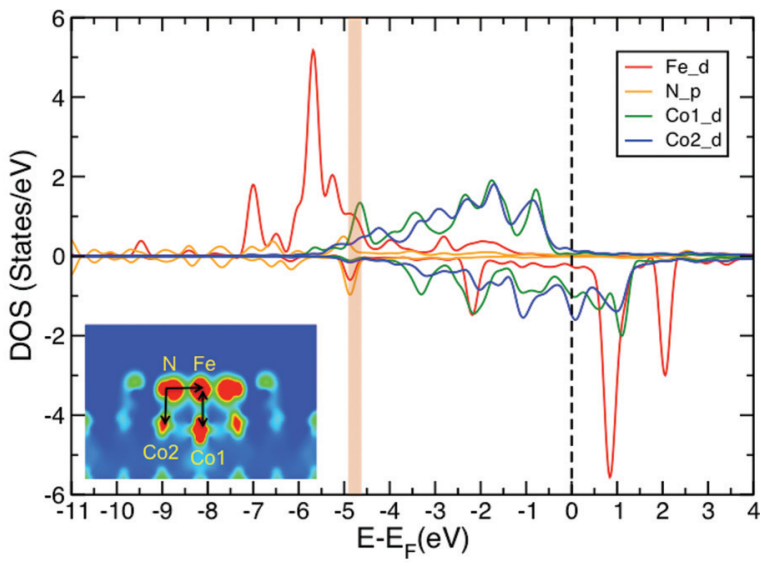

(b)

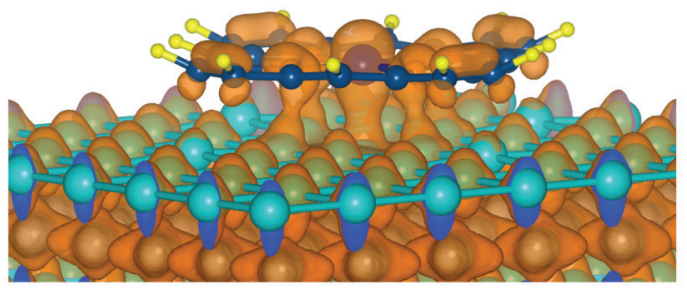

(c)

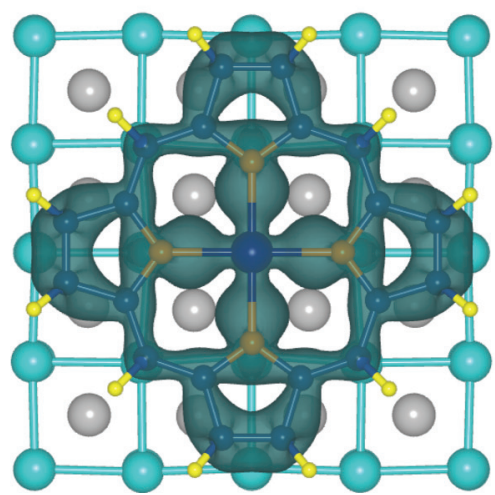

FIG. 7. (Color online) (a) Spin-polarized atom and orbitalresolved DOS for $\mathrm{FeP}$ on $\mathrm{Co}(001)$ in the TOP position. In the inset, a charge density cut in the (001) plane is shown for an energy window indicated as the shaded region in the DOS plot. Co1 and $\mathrm{Co} 2$ are the atoms of the Co substrate, situated just below $\mathrm{Fe}$ and $\mathrm{N}$ atoms of $\mathrm{FeP}$, respectively. (b) Side view of the magnetization density isosurface shown for the same energy interval to show the out-of-plane orbitals. (c) Top view of the magnetization density to show the in-plane orbitals taking part in the exchange interaction between $\mathrm{FeP}$ and the Co substrate.

to the Co substrate is about a factor of 4 larger than for the $\mathrm{Ni}$ substrate for (001) surface orientation. Among the Ni surface orientations, the (110) surface shows the weakest coupling with FeP. To summarize, irrespective of chemical species and orientation, all surfaces show FM coupling with FeP.

Apart from the exchange energies, the relative stabilities are also indicated in Table I through the total energies with respect to the ground state energy for each surface type and orientation. It is clearly observed that the most stable configuration is the

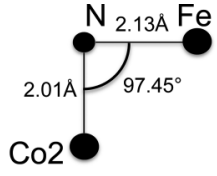

(a) $E^{e x}=0.39 \mathrm{eV}$

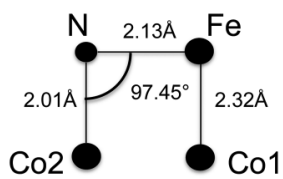

(d) $E^{e x}=0.39 \mathrm{eV}$

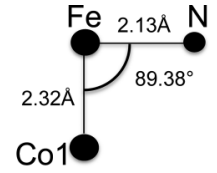

(b) $E^{e x}=0.55 \mathrm{eV}$

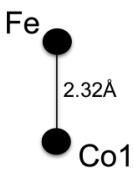

(e) $E^{e x}=0.67 \mathrm{eV}$

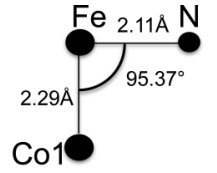

(c) $E^{e x}=0.69 \mathrm{eV}$

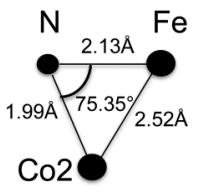

(f) $E^{e x}=0.49 \mathrm{eV}$
FIG. 8. Structural models extracted from fully optimized DFT calculations of FeP on different substrates indicated in the text. The geometries were not optimized further for these models which are used to calculate the total energies for FM and AFM alignments between Fe and Co moments. The energy differences between AFM and FM alignments are shown for each model, where +ve value of $E_{\text {ex }}$ means ferromagnetic coupling.

HOLLOW position for both $\mathrm{Co}$ and $\mathrm{Ni}(001)$ surfaces, whereas the TOP-R configuration is the most stable one for a $\mathrm{Ni}(110)$ surface. As mentioned before, stability is primarily dictated by the chemical bonds between the atoms in the molecule and in the first surface layer. Also, a difference in behavior in the relative stabilities between the Co and the Ni surface is observed due to the difference in number of $d$ electrons and hence the strength of bonding.

In order to have a better understanding of the exchange mechanism, we have performed several total energy calculations with the structural models extracted from the optimized geometries in our full DFT calculations. We have chosen the relevant parts of the optimized geometries to build the structural models, which are shown in Fig. 8 for the TOP configuration. This configuration was selected since it represents the case with most complex exchange interactions, as we shall see below. However, we will also present a model case from the HOLLOW configuration, below. As we have already stated, there are two kinds of surface-to-molecule exchange interactions, viz., (Co/Ni)-Fe direct exchange and (Co/Ni)-N-Fe indirect superexchange. Figure 8(a) represents the indirect superexchange between $\mathrm{Fe}$ and $\mathrm{Co}$ via $\mathrm{N}$, whereas Fig. 8(b) represents the Fe-Co direct exchange. Here Co1 $(\mathrm{Co} 2)$ is the Co atom just below $\mathrm{Fe}(\mathrm{N})$. As mentioned earlier, a rotation by $45^{\circ}$ decreases the distance between $\mathrm{Fe}$ and $\mathrm{Co}$. Figure 8(c) mimics the situation having a direct exchange but with a smaller Fe-Co distance. Figure 8(d) represents a model that includes both direct and indirect exchange, while Fig. 8(e) represents Fe-Co direct exchange in the absence of N. Figure 8(f) is taken from the optimized HOLLOW configuration. In this case, the Fe-N-Co angle is not close to $90^{\circ}$ but $75.35^{\circ}$ due to the geometry of the surface underneath. Based on our calculations, we can conclude that both direct and indirect exchange interactions are important to consider with varying weight based on particular situations of chemisorption and that both terms contribute with a ferromagnetic coupling. 


\section{Spin dipole contribution}

Although the spin moment remains similar in all chemisorbed situations, a deformation in the spin density is expected to be quite high for a low-symmetry structures, leading to a large value of the spin dipole moment $\left\langle T_{z}\right\rangle^{1}$ where $\left\langle T_{z}\right\rangle$ is the expectation value of the $z$ component of the spin-dipole operator $T$. Moreover spin densities differ due to different ligand fields exerted on FeP by different surfaces and their orientations. The discussion of the spin dipole moment is quite relevant for XMCD measurements, where the measured effective moment contains both spin and spin dipole moments. We have calculated the spin dipole moments by following the method suggested in Refs. 15 and 16. The calculated values of $7\left\langle T_{z}\right\rangle$ are $0.82,1.08$, and $0.79 \mu_{B}$ for FeP on $\mathrm{Co}(001), \mathrm{Ni}(111)$, and $\mathrm{Ni}(110)$ surfaces, respectively. It is clear from the signs and values of $\left\langle T_{z}\right\rangle$ that the effective spin moment defined as $S_{\text {eff }}=2\left\langle S_{z}\right\rangle+7\left\langle T_{z}\right\rangle$, can be highly affected by $\left\langle T_{z}\right\rangle$.

\section{Detection of spin state change}

Besides XAS and the spin dipole moments measured in XMCD experiments, we propose two additional experimental techniques to detect the change in the spin state of FeP, (i) Raman spectroscopy and (ii) spin-polarized STM (SP-STM). We shall first discuss our results for Raman intensities. For these calculations, we have considered the optimized geometries of FeP in both chemisorbed and physisorbed situations in the presence of the substrates and have calculated the vibrational normal modes of FeP in those optimized geometries only in the gas phase. The GAUSSIAN 09 program $^{17}$ was used at the B3LYP level of theory with the $6-31+\mathrm{G}(d, p)$ basis set. In these calculations, the geometries were taken from the optimized structures of $\mathrm{FeP}$ on $\mathrm{Co}(001)$ for chemisorbed and physisorbed situations. The calculated wave numbers above $1000 \mathrm{~cm}^{-1}$ were scaled using the correction factor $0.9614 .^{18}$ Raman intensities were calculated using the expression ${ }^{19}$

$$
I\left(v_{i}\right)=\frac{f S_{i}\left(v_{0}-v_{i}\right)^{4}}{v_{i}\left(1-e^{\frac{-h c v_{i}}{k_{B} T}}\right)},
$$

where $f$ is a scaling factor (1.0 in this case), $S_{i}$ are the calculated Raman activities, $v_{0}$ is the wave number corresponding to the excitation laser line in $\mathrm{cm}^{-1}$ (we used $457.9 \mathrm{~nm}$ as it is in the range used to measure the resonance Raman spectrum of porphyrins $\left.{ }^{20}\right), v_{i}$ is the calculated vibrational frequency, $h, c$, and $k_{B}$ are Planck's constant, speed of light, and Boltzmann's constant, respectively, while $T$ is the room temperature (293 K).

The Raman spectra of the triplet ( $S=1$ /physisorbed) and quintuplet ( $S=2 /$ chemisorbed) spin states differ substantially both in the peak intensity and peak position, as evident from Fig. 9. There are two spectral windows where these differences are significantly observed. First of all, the most intense peaks of the FeP (both in the case of the chemisorbed and physisorbed molecule) appear in the range of $110-450 \mathrm{~cm}^{-1}$, shown in the upper panel of Fig. 9. The highest band in this spectral window corresponds to a breathing vibration of the entire molecule and it is shifted to lower wave numbers in the case of the chemisorbed FeP as compared to the physisorbed one by approximately $35 \mathrm{~cm}^{-1}$. A shift of this magnitude
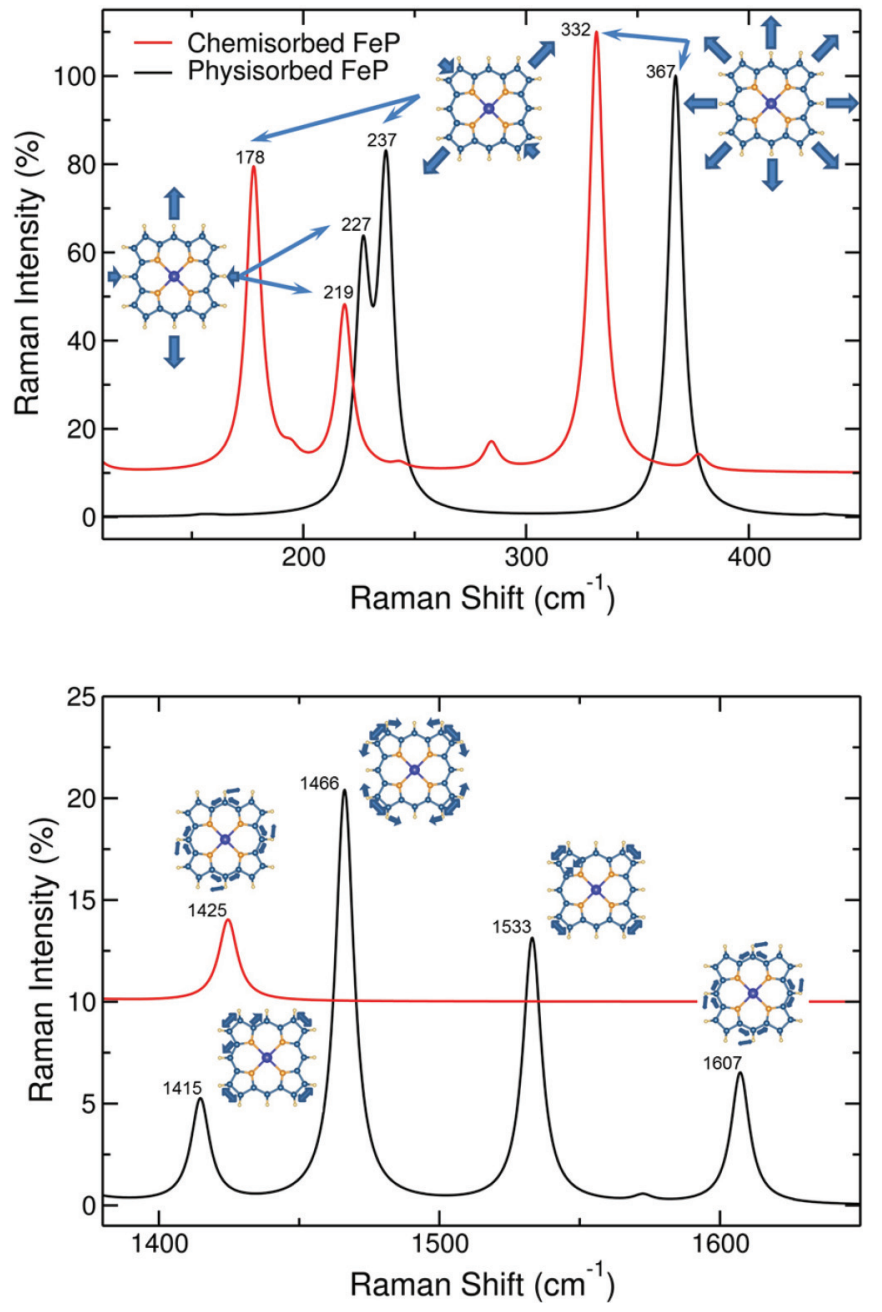

FIG. 9. (Color online) Calculated Raman intensities for FeP in chemisorbed (red lines) and physisorbed (blue lines) geometries. In the upper panel, Raman intensities are shown for shifts in the range $110-450 \mathrm{~cm}^{-1}$, whereas the lower panel contains wave numbers in the range $1380-1650 \mathrm{~cm}^{-1}$. In both panels, the vibrational modes corresponding to the peaks are denoted along with the corresponding wave numbers.

should easily be observed in Raman experiments. ${ }^{21}$ A shift of even larger magnitude occurs in the band at $237 \mathrm{~cm}^{-1}$ of the physisorbed FeP. It is assigned to a symmetric stretching vibration of the entire molecule and it appears $60 \mathrm{~cm}^{-1}$ lower in the chemisorbed situation. The third major difference in this spectral window is the inversion of the two lower Raman peaks. The less intense peaks correspond to the two spectra representing diagonal symmetric stretching vibrations of the entire molecule. In the range of $1380-1650 \mathrm{~cm}^{-1}$ (shown in the lower panel of Fig. 9), three peaks occur with relatively high intensity in the physisorbed FeP that have correspondence in the chemisorbed molecule at much lower wave numbers. For instance, the physisorbed $\mathrm{FeP}$ band at $1607 \mathrm{~cm}^{-1}$ corresponds to the same $\mathrm{C}-\mathrm{C}-\mathrm{C}$ asymmetric stretching coupled with $\mathrm{C}-\mathrm{H}$ bending that is assigned to the $1425 \mathrm{~cm}^{-1}$ chemisorbed FeP band. The other two peaks are assigned to in-plane C-C stretching and $\mathrm{C}-\mathrm{N}-\mathrm{C}$ bending vibrations. Thus, this spectral window would be ideal to experimentally detect the switching 
(a)
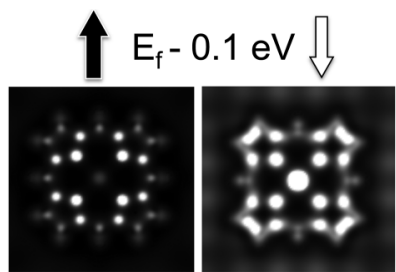

(b)
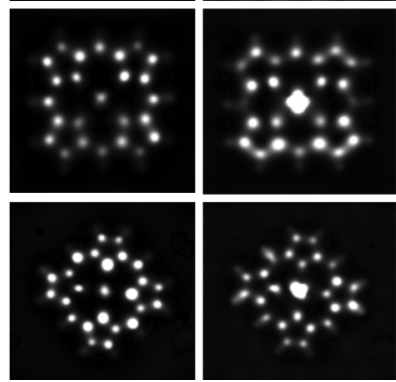

\section{CONCLUSIONS}

In summary, we have demonstrated by ab initio density functional calculations that chemisorption of an $\mathrm{FeP}$ molecule on magnetic substrates of different species and orientations can switch the spin state of the molecule from $S=1$ to $S=2$. In particular, we demonstrate that this happens for $\mathrm{FeP}$ chemisorbed on $\mathrm{Co}$ and $\mathrm{Ni}(001)$ as well as $\mathrm{Ni}(110)$ and $\mathrm{Ni}(111)$ surfaces. This change in spin state is brought by an increase in the $\mathrm{Fe}-\mathrm{N}$ bond lengths in $\mathrm{FeP}$, induced by the strong covalent interactions between the chemisorbed molecule and the substrate atoms. The ground state geometries correspond to the optimization of the chemical bonds between $\mathrm{N}$ atoms of $\mathrm{FeP}$ and the underlying substrate atoms. The signature of chemisorption is evident from the broadening of the spectroscopic features in measured $\mathrm{N} K \mathrm{XA}$ and $\mathrm{Fe} L_{2,3} \mathrm{XMCD}$ spectra on $\mathrm{Ni}$ in contrast to sharp multiplet-like features in the case of adsorption on a $\mathrm{Cu}$ substrate. ${ }^{5}$ Moreover, we have analyzed the mechanisms of exchange interaction by simple structural models constructed from our DFT calculations and demonstrated that both superexchange and direct exchange are operational in mediating a ferromagnetic interaction between $\mathrm{Fe}$ and the substrate magnetic atoms. Our calculations show that the exchange interaction between $\mathrm{FeP}$ and the magnetic substrate is almost 4 times less on a Ni substrate compared to a Co substrate. Finally, we show that it is possible to detect the change in the spin state of FeP due to chemisorption by XMCD and Raman spectroscopy along with different features in spin-polarized scanning tunneling microscopy for different spin channels.

\section{ACKNOWLEDGMENTS}

B.S. gratefully acknowledges financial support from Carl Tryggers Stiftelse and Swedish Research Council (VR). O.E. is grateful to the KAW foundation, eSSENCE, and the ERC (project 247062 - ASD) for support. P.P. would like to acknowledge membership of the UK's HPC Materials Chemistry Consortium, which is funded by EPSRC (Grant No. EP/F067496). We also acknowledge SNIC-UPPMAX, SNIC-HPC2N, and SNIC-NSC centers under the Swedish National Infrastructure for Computing (SNIC) resources for the allocation of time in high performance supercomputers.

\footnotetext{
*Biplab.Sanyal@physics.uu.se

${ }^{1}$ S. Bhandary, S. Ghosh, H. Herper, H. Wende, O. Eriksson, and

B. Sanyal, Phys. Rev. Lett. 107, 257202 (2011).

${ }^{2}$ T. A. Jung, R. R. Schlitter, J. K. Gimzewski, H. Tang, and C. Joachim, Science 271, 181 (1996); A. R. Rocha, V. M. GarciaSuarez, S. W. Bailey, C. J. Lambert, J. Ferrer, and S. Sanvito, Nat. Mater. 4, 335 (2005); J. V. Barth, Annu. Rev. Phys. Chem. 5, 375 (2007); M. Mannini, F. Pineider, P. Sainctavit, C. Danieli, E. Otero, C. Sciancalepore, A. M. Talarico, M.-A. Arrio, A. Cornia,

D. Gatteschi, and R. Sessoli, Nat. Mater. 8, 194 (2009).

${ }^{3} \mathrm{H}$. Wende et al., Nat. Mater. 6, 516 (2007).

${ }^{4}$ M. Bernien et al., Phys. Rev. Lett. 102, 047202 (2009).

${ }^{5}$ H. C. Herper et al., Phys. Rev. B 87, 174425 (2013).
}

${ }^{6}$ M. Bernien, X. Xu, J. Miguel, M. Piantek, Ph. Eckhold, J. Luo, J. Kurde, W. Kuch, K. Baberschke, H. Wende, and P. Srivastava, Phys. Rev. B 76, 214406 (2007).

${ }^{7}$ J. Brede, N. Atodiresei, S. Kuck, P. Lazic, V. Caciuc, Y. Morikawa, G. Hoffmann, S. Blügel, and R. Wiesendanger, Phys. Rev. Lett. 105, 047204 (2010).

${ }^{8}$ Md. E. Ali, B. Sanyal, and P. M. Oppeneer, J. Phys. Chem. C 113, 14381 (2009).

${ }^{9}$ G. Kresse and J. Hafner, Phys. Rev. B 47, R558 (1993).

${ }^{10} \mathrm{G}$. Kresse and J. Furthmüller, Phys. Rev. B 54, 11169 (1996).

${ }^{11}$ V. I. Anisimov, F. Aryasetiawan, and A. I. Lichtestein, J. Phys.: Condens. Matter 9, 767 (1997). 
${ }^{12}$ P. M. Panchmatia, B. Sanyal, and P. M. Oppeneer, Chem. Phys. 343, 47 (2008).

${ }^{13}$ S. Grimme, J. Comput. Chem. 27, 1787 (2006).

${ }^{14}$ C. S. Guo, L. Sun, K. Hermann, C. F. Hermanns, M. Bernien, and W. Kuch, J. Chem. Phys. 137, 194703 (2012).

${ }^{15}$ R. Wu and A. J. Freeman, Phys. Rev. Lett. 73, 1994 (1994).

${ }^{16}$ T. Oguchi and T. Shishidou, Phys. Rev. B 70, 024412 (2004).

${ }^{17}$ GAUSSIAN 09, Revision A.1, M. J. Frisch et al., Gaussian, Inc., Wallingford, CT, 2009.
${ }^{18}$ D. C. Young, Computational Chemistry: A Practical Guide for Applying Techniques to Real-World Problems (John Wiley, New York, 2001).

${ }^{19}$ L. Padmaja et al., Spectrochim. Acta Part A 71, 252 (2008).

${ }^{20}$ G. A. Schick and Ziqi Sun, Thin Solid Films 248, 86 (1994); K. Nakamoto, Coord. Chem. Rev. 226, 153 (2002); C. De Silva et al., Inorg. Chim. Acta 287, 21 (1999).

${ }^{21}$ R. Aroca, N. Pieczonka, and A. P. Kam, J. Porphyrins Phthalocyanines 5, 25 (2001).

${ }^{22}$ J. Tersoff and D. R. Hamann, Phys. Rev. B 31, 805 (1985). 